[Aus dem Institut für gerichtliche Medizin der Unirersität Graz.] (Vorstand: Prof. J. Kratter.)

\title{
Zur Kenntnis der agglutinierenden Wirkung von Rückständen normalen Menschenharnes. ${ }^{1}$
}

\author{
II. Mitteilung. \\ Von \\ Dr. Hermann Pfeiffer, \\ Privatdozent.
}

$\mathrm{Da}$ die hier wiederzugebenden Versuche aus äuBeren Gründen abgebrochen werden muBten und die daraus sich ergebenden Resultate in absehbarer Zeit bis in ihre letzten Konsequenzen nicht verfolgt werden können, so sehe ich mich genötigt, darüber heute schon zu berichten, wohl wissend, daß damit eine völlige Klärung der sich hier aufrollenden Fragen nicht erzielt werden konnte.

In einer ersten Mitteilung wurde darauf hingewiesen, daB es durch entsprechende Vorbehandlung gelingt, normalen Menschen- und Tierharn, der genuin und nach Absättigung seiner freien Säure den Erythrozyten gegenüber sich völlig indifferent verhält, derart zu verändern, daß die nun gewonnene Flüssigkeit, abgesehen von anderen, im Tierexperimente wohl $\mathrm{zu}$ charakterisierenden Giftwirkungen, auBerordentlich intensive agglutinierende Eigenschaften zu äuBern vermag. Es wurde ferner nachgewiesen, daB diese Wirkung zurückgeführt werden müsse auf einen Körper kolloidaler Natur, der schon bei gewöhnlicher Temperatur, noch mehr aber bei höheren Wärmegraden sehr hinfällig ist, so daB in relativ kurzer Zeit aktive Rūckstände ihre Wirksamkeit ganz oder doch fast vollkommen einbüßen. Es wurde gezeigt, daB eine elektive Absättigung und

1 Ausgeführt mit Unterstützung der k. Akademie der Wissenschaften in Wien aus dem Legate Wedl. 
Bindung der als Harnagglutinin (H.-A.) bezeichneten Substanz durch Vorbehandlung mit Erythrozyten möglich sei, eine Erfahrung, die in Übereinstimmung mit dem eben Erwähnten für das Vorhandensein einer haptophoren Gruppe und für das Vorliegen eines Antigens zu sprechen schien. Im Gegensatze dazu konnte aber durch Vorbehandlung von Tieren (Kaninchen) eine Antikörperbildung in ihrem Serum nicht beobachtet werden. Endlich konnte mit großer Wahrscheinlichkeit dargetan werden, daB die in Rede stehende Substanz aus einer weder hemmend noch agglutinierend wirkenden, also indifferenten, schwer dialysablen Vorstufe des Harnes entstehe. Dabei muBte die Erfahrung gemacht werden, daB unter sonst gleichen äußeren Versuchsbedingungen die Ausbeute an wirksamem Prinzipe einem unverständlichen Wechsel unterworfen war, indem verschiedene Harnproben eines und desselben Menschen bei gleichbleibender Lebensweise das eine Mal eine auBerordentlich intensive, das andere Mal aber eine kaum nachweisbare Wirkung zeigten. Es wurde endlich noch besonders darauf hingewiesen, daß dann und wann ganz frisch bereitete Rückstände in konzentrierten Lösungen sich als unwirksam erwiesen, während in höheren Verdünnungsgraden das Agglutinationsphänomen in die Erscheinung trat. Die Hemmungszone derartig reagierender Rückstände verliert sich kurze Zeit nach der Darstellung spontan, wobei gleichzeitig ein Hinausrücken der oberen Wirkungsgrenze zu beobachten ist. Unter dem Vorbehalt, daB es gelänge, den Nachweis des tatsächlichen Vorliegens eines Agglutinoides $\mathrm{zu}$ erbringen, wurde die in Rede stehende Beobachtung damals in der Weise hypothetisch erklärt, daB die Hemmungszone konzentrierterer Lösungen durch eine Konkurrenz eines avideren, seiner agglutinophoren Gruppe beraubten Agglutinoides bei Erhaltenbleiben seiner haptophoren Gruppe mit einem weniger aviden, mit beiden Gruppen ausgestatteten Agglutinin wahrscheinlicherweise zustande komme, Diese Deutung der Sachlage konnte damals nur mit Wahrscheinlichkeit erfolgen, da der strikte Beweis des Vorliegens eines Agglutinoides noch nicht erbracht war und im Hinblick auf ein ähnliches, zuerst von Landsteiner und Jagič bei Lösungen kolloidaler Kieselsäure beobachtetes Phänomen derartige, zur Annahme einer hemmenden Modifikation drängende Fehlerquellen a priori nicht ausgeschlossen werden konnten. Eine recht weitgehende Analogie des Wirkungsmechanismus dieser anorganisehen Kolloide mit dem hier in Rede stehenden Körper, ebenso wie die wechselnden quantitativen Resultate bei scheinbar gleich bleibenden äußeren Versuchsbedingungen ließen es nun wünschenswert erscheinen, ihnen experimentell etwas näher zu treten. Namentlich schien ein Studium der stark wechselnden Befunde in der Aktivität solcher Rückstände sowie der Hemmungszone zur Klärung der Sachlage nötig. 
I.

Sprachen auch manche der hier in Kürze wiedergegebenen früheren Beobachtungen dafür, daB es sich bei dem Auftreten der agglutinierendeu Wirkung um Umlagerungen im Moleküle eines kompliziert gebauten Körpers handeln dürfte, so überraschten andererseits wieder die weitgehenden Analogien mit den von Landsteiner und Jagic beschriebenen Ausflockungserscheinungen ron zelligen Elementen und von Eiweißlösungen durch anorganische Kolloide und forderten dazu anf, zu untersuchen, ob nicht vielleicht das in Rede stehende Phänomen auf derartige Ursachen zurückgeführt werden müsse.

In kolloidalem Zustande zeigen anorganische Körper (Kieselsäure, Neutralsalze) das Agglutinationsphänomen Blutkörperchen und anderen Zellen gegenüber, nach ihrem Zusatz zu EiweiBlösungen tritt zunächst eine Trübung, dann eine Ausflockung auf. Wie bei dem H.-A., so konnte auch dort von einer Spezifität der Wirkung im Sinne ihrer Wirksamkeit nur gegen bestimmte Blutkörperchenarten, wie dies z. B. bei manchen Schlangengiften beobachtet wurde, nicht die Rede sein. Alle bisher untersuchten Blutarten erwiesen sich annähernd gleich empfindlich gegen aktive Rückstände (R.) ebenso wie anorganische Kolloide.

Auch Landsteiner und Jagič beschrieben die Erscheinung der Hemmung durch einen Überschuß ihrer Kolloide, während verdünnte Lösungen lebhaft fällende Kraft zeigten. Sie betonen, daB im Gegensatz $z u$ der bei organischen Agglutininen erkennbaren relativen Stabilität gegen schädigende Einflüsse verschiedenster Art ihre aktiven Lösungen schon durch Stehen spontan rasch unwirksam werden, wie dies auch für das H.-A. beobachtet wurde. Und endlich: Hier wie dort konnte durch Vorbehandlung der Versuchstiere (Kaninchen) Antikörperbildung nicht nachgewiesen werden.

$\mathrm{Zu}$ einer Analogisierung auf dieser Basis als ungeeignet mußte von vorneherein die Nichtspezifität der Wirkung angesehen werden, da auch organische Agglutinine - ich habe hier speziell das Abrin im Auge! -, so weit heute die Erfahrungen reichen, eine ganz allgemeine, also gleichfalls nicht spezifische Wirkung äußern und von der Spezifität zur Nichtspezifität fließende Übergänge zu beobachten sind, so daß sich aus diesem Umstand ein Unterscheidungsmerkmal zwischen organischen und anorganischen Agglutininen nicht ableiten läßt.

Auch die Tatsache des Auftretens von Trübungen in klaren Eiweiblösungen durch den $R$. darf in dem ebenerwähnten Sinn nicht herangezogen werden, da erst kürzlich Michaelis und Steindorf, ferner auch Landsteiner und Jagič nach dem Zusatz von organischen Agglutininen, 
deren Antigencharakter nachgewiesen ist, wie beim Abrin und Ricin, Analoges beschrieben haben.

Da, wie oben erwähnt, genuiner Harn nach Abstumpfung seiner freien Säure sich indifferent verhältt ${ }^{1}$, also weder eine agglutinierende noch auch hemmende Wirkung zeigt, so war in Anbetracht der angeführten Analogien die Mōglichkeit gegeben, es gehen bei der Darstellung - also bei Vakuumdestillation unter Alkoholzusatz und beim Erhitzen - ein oder mehrere anorganische Harnbestandteile in den kolloidalen Zustand über und bedingen nun das Agglutinationsvermögen des R. Diese Möglichkeit konnte dann ausgeschlossen werden, wenn sich experimentell zeigen lieB, daB aus dialysiertem Harne gleichfalls aktive Rückstände gewonnen werden können. Es mußten bei dieser Versuchsanordnung alle Fehlerquellen dieser Art wegfallen. Tatsächlich gelang es nun, wie in der ersten Mitteilung gezeigt, aber nicht genügend betont wurde, in dem in Bd. LIV dieser Zeitschrift, S. 453 mitgeteilten Versuche aus früher dialysiertem Harn durch Erhitzen agglutinierende R. in derselben Weise zu erhalten, wie wenn dieses Reinigungsmanöver vorher nicht durchgeführt worden war.

Diese Beobachtung beweist, daß es sich bei der Fntstehung der agglutinierenden Wirkung nicht um die anorganischen Kolloide Landsteiners und Jagič' in dem oben erwähnten Sinne handeln könne, daß vielmehr schon das indifferente Ausgangsmaterial, die Vorstufe des H.-A., ein Kolloid sein müsse.

Dieser Umstand veranlaßte mich früher schon, hypothetisch die Ansicht auszusprechen, es könne sich hier vielleicht um das von Sehattenfroh als Lysogen beschriebene, von Abderhalden und Pregl als EiweiBabkömmling chemisch definierte Kolloid handeln.

Auf eine zweite, gleichfalls von vorneherein nicht absolut auszuschließende und bisher nicht in Betracht gezogene Fehlerquelle hat gesprächsweise Landsteiner aufmerksam gemacht, indem er gleichzeitig den experimentellen Weg wies, über ihr Vorliegen zu entscheiden.

Die Darstellung der aktiven $R$. sowohl, wie auch die Erhitzung der Harnproben auf $80^{\circ}$ war bisher immer und ausschlieBlich in Glasgefäßen erfolgt.

Es wäre nun möglich gewesen, daB aus den Gefäßwandungen im Verlaufe der oft Stunden währenden Versuche Spuren von Silikaten in

1 Vgl. dazu die Angaben von Sabrazès and Fauquet (Compt. rend. de la Soc. de Biol., 1901), sowie von Cannus und Pagniez (Ebenda, 1901) über die hämolytische Wirkung des Harnes, die von $\mathrm{Pugnat}($ Ebenda, 1901) anf Anisotonie zurïck. geführt wird. 
kolloidaler Form in die Flüssigkeit übergehen und Im Rückstand das beschriebene Phänomen veranlassen. Sollte auch diese Fehlerquelle ausgeschlossen werden, so durften beim Erhitzen in PlatingefäBen - eine Darstellung im Vakuum war aus naheliegenden Gründen undurchführbar! - aktire R. nicht beobachtet werden. Es gelang nun in zahlreichen Versuchen, deren ausführliche Wiedergabe hier wohl unterbleiben kann, durch Erhitzen verschiedener Harnproben in der Menge von je $10 \mathrm{ccm}$ in Platingefäßen während eines Zeitraumes von $30^{\prime}$ bis zu 2 Stunden gleichfalls kräftig agglutinierende Rückstände ganz analog den in T'abelle XII der ersten Mitteilung beschriebenen zu erhalten. So darf auch dieser Einwand als entkräftet angesehen werden.

\section{II.}

Es verdiente weiterhin die Beobachtung eine eingehendere Prüfung, daß verschiedene Proben des Harnes ein und derselben Versuchsperson in kurzen zeitlichen Zwischenräumen eine weitgehende Differenz in ihrem Agglutinationsvermögen erkennen ließen, was aus den auszugsweise in Tabelle $\mathrm{V}$ der ersten Mitteilung wiedergegebenen Resultaten gefolgert und durch zahlreiche neue Erfahrungen vielfach bestätigt werden konnte.

Es wurden aus dem gleichmäBigen Materiale des Morgenharnes einer Versuchsperson und bei scheinbar gleichbleibender Darstellungsmethodik in bunter Reihenfolge einmal stark, das andere Mal schwach wirksame, endlich aber auch unwirksame $R$. gewonnen.

Diese Erscheinung konnte durch zwei Ursachen bewirkt werden:

1. durch einen starken quantitativen Wechsel in der Ausscheidung der kolloidalen Vorstufe durch die Nieren;

2. im Hinblick auf die Hinfälligkeit der wirksamen Erscheinungsform des Körpers durch nicht eliminierte oder nicht eliminierbare Differenzen in der Darstellungsmethodik, die unter gegebenen Umständen schon schwere Schädigungen der Wirksamkeit nach sich ziehen muBten.

Beide Möglichkeiten zu prüfen, schien um so wichtiger, als eine Fruchtbarmachung dieses Phänomens für die Physiologie oder Pathologie eine verläBliche quantitative Ausbeute zur unerläBlichen Vorbedingung haben mußte. Für eine Entscheidung dieser Fragen lieferte einen Fingerzeig die groBe Wahrscheinlichkeit der Identität des nicht dialysablen Eiweißabkömmlings ron $A b d e r h a l d e n$ und Pregl mit der gleichfalls nicht dialysablen, indifferenten Vorstufe der aktiven R. Der Hinblick auf diese wahrscheinliche Identität konnte für die Arbeitsrichtung insofern ron Bedeutung sein, als die erste der früher aufgestellten Möglichkeiten in dem Momente an Wahrseheinlichkeit verlor, wo nachgewiesen werden konnte, dab der 
Abderhalden-Preglsche Körper beim Gesunden in konstanten Mengen zur Ausscheidung komme. Unveröffentlichten Versuchen von H. Eppinger und Pregl über die quantitativen Ausscheidungsverhältnisse beim Gesunden und Kranken entnehme ich nun die Erfahrung, daß dieser Körper unter physiologischen Verhältnissen in relativ konstanten Mengen im Harne erscheine. Demnach muBte von vorneherein die zweite der angeführten Möglichkeiten in hohem MaBe in Betracht gezogen werden. Darüber, ob sie sich zur Tatsache werde erbärten lassen, konnten unter Auswertung des agglutinierenden Titres angestellte Parallelversuehe mit Harnproben derselben Provenienz bei Variationen der Darstellungsmethodik Aufsehlub geben.

Zunächst wurde die zufällige Erfahrung verfolgt, daB ein und derselbe Harn, von dem eine Probe im Vakuum, die andere auf dem Wasserbade bei $80^{\circ} \mathrm{C}$ behandelt worden war, wesentliche Differenzen seiner Wirksamkeit zeigte, was Tabelle I erläutern möge.

Alle im folgenden wiedergegebenen Versuche wurden, wo dies nicht ausdrücklich anders hervorgehoben ist, mit einer 5 prozentigen Aufsehwemmung von Meerschweinchenerythrozyten nach den allgemein gebräuchlichen Regeln des Reagensglasversuches angestellt. Die auf dem Wasserbade erhitzten Harne wurden nach Absättigung ihrer freien Säure mit Kochsalzlösung auf ihr früheres Volumen gebracht, die im Vakuum eingeengten Proben in einer Konzentration verwendet, dab $1^{\mathrm{ccm}}=5^{\mathrm{cem}}$ des Ausgangsmateriales entsprach. In den Tabellen wurden, um quantitative Vergleiche der Wirksamkeit durchführen zu können, der. Agglutinationstitre der Vakuumrückstände immer auf die Konzentration des Ausgangsmateriales umgerechnet. Zur Verdeutlichung der Stärke des eingetretenen Effektes wurden folgende Bezeichnungen und Abkürzungen gewählt:

$$
\left.\begin{array}{rl}
\mathrm{m} . & =\text { maximale } \\
\mathrm{fm} . & =\text { fast maximale } \\
\mathrm{sd} . & =\text { sehr deutliche } \\
\mathrm{d} . & =\text { deutliche } \\
\mathrm{Sp} . & =\text { spurenweise }
\end{array}\right\} \text { Agglutination. }
$$

Tabelle I.

\begin{tabular}{c|c|c}
\hline Menge auf Harn berechnet & Vakuumdestillation & $\begin{array}{c}\text { Optimum bei Erhitzung } \\
\text { auf } 80^{\circ}\left(60^{\prime}\right)\end{array}$ \\
\hline 1.0 & $\mathrm{~m}$. & $\mathrm{m}$. \\
0.5 & $\mathrm{~m}$. & fm. \\
0.25 & $\mathrm{~m}$. & sd. \\
0.13 & $\mathrm{~m}$. & $\mathrm{d}$. \\
0.06 & $\mathrm{m.}$ & - \\
0.03 & $\mathrm{~m}$. & - \\
0.015 & $\mathrm{sd}$. & - \\
0.007 & Sp. & - \\
0.004 & - & - \\
0.002 & - &
\end{tabular}


Aus dieser Tabelle und aus zahlreichen anderen hier nicht näher wiederzugebenden Versuchen geht hervor: Trotzdem auch die Erhitzung auf $80^{\circ} \mathrm{C}$ zu aktiven $R$. führt, so liefert doch im allgemeinen die Darstellung im Vakuum weitaus wirksamere Lösungen.

Angesichts dieser Tatsache war es wichtig, festzustellen, welche Faktoren denn die Bildung wirksamer $R$. begünstigen. Von solchen muBten in Betracht gezogen werden: die Temperatur, der Gehalt des Ausgangsmateriales an freier Säure, daher auch die Menge und die Konzentration des Harnes, die Versuchsdauer und endlich die Wirkung des zur Vakuumdarstellung verwendeten Alkohols.

Es muBte daher zuerst entschieden werden, bei welcher Temperatur unter sonst gleichen Versuchsbedingungen die wirksamsten R. erhalten werden. Darüber belehrte der folgende Versuch:

Von 16 Harnproben wurden je 4 bei $40^{\circ}$, je 4 bei $60^{\circ}$, ebensoviele bei $80^{\circ}$ und endlich dieselbe Anzahl bei $100^{\circ} \mathrm{C}$, während der in der Tabelle II ersichtlichen Zeiten in der Menge von je $10^{\mathrm{cm}}$ gehalten, die freie Säure abgesättigt, die Lösungen durch Zusatz von Wasser auf das frühere Volumen gebracht, bzw. das bei $100^{\circ}$ abgespaltene Ammoniak neutralisiert und nun die Proben auf ihre Wirksamkeit geprüft. Eine Parallelprobe im Vakuum unter Alkoholzusatz ergab einen sehr kräftig agglutinierenden R., während von den erhitzten Proben nur die auf $80^{\circ}$ erwärmten sich wirksam erwiesen.

Es zeigte sich also, daß während der angegebenen Versuchszeiten bei $40^{\circ}$ und $60^{\circ}$ H.-A. nicht erhalten werden konnte, während bei $80^{\circ}$ nach einem 90 Minuten dauernden Versuche die kräftigsten Lösungen zu beobachten waren. Bei $100^{\circ}$ konnte auch in diesen Versuchen, ganz analog den in der ersten Mitteilung veröffentlichten, eine Agglutininbildung in keiner Zeitperiode beobachtet werden. Bei Wiederholung dieser Versuehe wurde ferner in der Weise vorgegangen, daB einerseits die Einwirkung von $40^{\circ}$ und $60^{\circ}$ bis zu 24 Stunden ausgedehnt, andererseits jene von $100^{\circ}$ schon nach 5 und 10 Minuten geprüft wurde, wobei dieselben negativen Resultate erhalten wurden.

Tabelle II.

\begin{tabular}{|c|c|c|c|c|c|c|c|c|c|c|c|c|c|c|c|c|c|}
\hline $\begin{array}{l}\text { Darstellung } \\
\text { bei: }\end{array}$ & \multicolumn{4}{|c|}{$40^{\circ}$} & \multicolumn{4}{|c|}{$60^{\circ}$} & \multicolumn{4}{|c|}{$80^{\circ}$} & \multicolumn{4}{|c|}{$100^{\circ}$} & $\begin{array}{c}40^{\circ} \\
\text { Vakuum }\end{array}$ \\
\hline $\begin{array}{l}\text { Zeit der } \\
\text { Einwirkg.: }\end{array}$ & 8 & & & 영 & 8 & : & 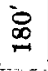 & 总 & 琚 & 8 & 8 & 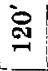 & is & के & 8 & : & \\
\hline 1.0 & - & - & - & - & - & - & - & - & - & m. & $\mathrm{m}$. & m. & - & -1 & - & - & m. \\
\hline 0.5 & - & - & - & - & $1-$ & - & - & - & - & d. & $\mathrm{m}$ & d. & - & - & & $1-$ & m. \\
\hline 0.25 & - & - & - & - & - & - & -1 & - & - & Sp. & m. & Sp. & - & - & -1 & - & m. \\
\hline 0.13 & - & - & - & - & - & - & -1 & -1 & - & -1 & sd. & -1 & - & -1 & -1 & - & m. \\
\hline 0.06 & - & - & - & - & - & - & - & - & - & - & d. & -1 & - & -1 & - & - & nl. \\
\hline 0.03 & - & - & - & - & - & - & - & - & - & - & $\mathrm{Sp}$ & - & - & -1 & - & - & $\mathrm{m}$. \\
\hline 0.015 & - & - & - & - & - & - & -1 & - & - & - & - & -1 & - & -1 & - & - & sd. \\
\hline 0.007 & - & - & -1 & - & - & - & - & - & - & -1 & - & - & - & -1 & $-!$ & - & d. \\
\hline$\emptyset$ & 一 & & $-i$ & -1 & - & - & - & - & - & - & -1 & - & - & - & -1 & -1 & - \\
\hline
\end{tabular}


Diese Versuche beweisen, daB das Optimum der Darstellung beim einfachen Erhitzen bei $80^{\circ}$ und bei einer Einwirkungsdauer von ca. 1 bis $1 \frac{1}{2}$ Stunden liegt, da $B$ unter diesen Versuchsbedingungen durch Temperaturen von 40,60 und $100^{\circ}$ H.-A. sich überhaupt nicht bildet, daB jedoch unter Alkoholzusatz im Vakuum bei $40^{\circ}$ weitaus die günstigsten Bedingungen zur Bildung des H.-A. gegeben sind. Daraus wieder kann gefolgert werden, daB die Wirkung des Alkohols für das Zustandekommen der agglntinierenden Wirkung als vorteilhaft - allerdings nur innerhalb gewisser Grenzen! - angesehen werden muß.

Was die Bedeutung der Menge des zur Darstellung verwendeten Harnes anlangt, so konnte durch einfaches Erhitzen verschiedengroßer Proben $\left(5 \mathrm{bis} 500^{\mathrm{com}}\right.$ ) auf $80^{\circ}$ die Beobachtung gemacht werden, daB bei Verwendung gröBerer Versuchsmengen unwirksame oder doch nur schwach wirksame R. erzielt wurden, und daB dabei der Zeitpunkt des Eintrittes der agglutinierenden Wirkung hinausgeschoben wurde, während kleine Mengen $\left(10^{\mathrm{ccm}}\right)$ desselben Materiales kräftig agglutinierende R. lieferten. Ähnliche Parallelversuche wurden auch unter Alkoholzusatz im Vakuum gemacht, wobei es sich zeigte, daB hier innerhalb gewisser Grenzen bei Verwendung größerer Mengen viel wirksamere R. gewonnen wurden.

Um über die Bedeutung der freien Sāure, bzw. des freien Alkali für das in Rede stehende Phänomen AufschluB zu erhalten, wurden, wie aus Tabelle III hervorgeht, verschiedene Proben unter Zusatz von Essigsäure und Kalilauge oder aber bei ihrer natürlichen saueren Reaktion bei Zimmertemperatur, bei $50^{\circ}$ und $80^{\circ}$ während der angegebenen Versuchszeiten gehalten, andere wieder auf gewöhnliche Weise im Vakuum behandelt.

Tabelle III.

\begin{tabular}{|c|c|c|c|c|c|c|}
\hline Temperatur: & \multicolumn{2}{|c|}{ Zimmertemperatur } & \multicolumn{4}{|c|}{$50^{\circ}$} \\
\hline Reaktion: & alkalisch & sauer & \multicolumn{2}{|c|}{ alkalisch } & \multicolumn{2}{|c|}{ sauer } \\
\hline $\begin{array}{c}\text { Zeit der } \\
\text { Einwirkung: }\end{array}$ & \multicolumn{2}{|c|}{36 Stunden } & $60^{\prime}$ & $120^{\prime}$ & $60^{\circ}$ & $120^{\prime}$ \\
\hline Agglutivation: & 6 & $\emptyset$ & - & schwach & - & schwaeh \\
\hline
\end{tabular}

\begin{tabular}{|c|c|c|c|c|c|c|c|}
\hline Temperatur: & \multicolumn{6}{|c|}{$80^{\circ}$} & \multirow{2}{*}{$\frac{40^{\circ} \text { Vakuum }}{\text { genuin }}$} \\
\hline Reaktion: & \multicolumn{2}{|c|}{ alkalisch } & \multicolumn{2}{|c|}{ sauer } & \multicolumn{2}{|c|}{ genuin } & \\
\hline $\begin{array}{c}\text { Zeit der } \\
\text { Einwirkung: }\end{array}$ & $30^{\prime}$ & $60^{\prime}$ & $30^{\prime}$ & $60^{\prime}$ & $30^{\prime}$ & $60^{\circ}$ & \\
\hline Agglutination & stark & dentlich & $\emptyset$ & $\emptyset$ & stark $<$ & sehr stark & $\begin{array}{c}a m \\
\text { stïrksten }\end{array}$ \\
\hline
\end{tabular}


Es zeigte sich also, daB auch hier durch das Vakuum die wirksamsten $R$. gewonnen wurden, da $B$ aber das Auftreten agglutinierender Eigenschaften bei alkalischer Reaktion auch bei $80^{\circ}$ beobachtet wurde, während nach 2 stündiger Einwirkung von $50^{\circ}$ sowohl sanere als alkalische Proben, wenn auch schwache, so doch immerhin nachweisbare Mengen des H.-A. darboten. Im Vergleiche mit den früher mitgeteilten Ergebnissen bei $60^{\circ}$ kann speziell aus dem letztgenannten Umstande gefolgert werden, daB für das Zustandekommen der agglutinierenden Wirkung auch niedrigere Hitzegrade als oben angegeben dann genügen, wenn sie bei extremer Reaktion einwirken, wenn also durch den ProzeB eine intensivere Schädigung - immer natürlich innerhalb gewisser Grenzen! - bedingt ist.

In ähnlichem Sinne dürften übrigens, auch die Ergebnisse gedeutet werden, daß eine Erwärmung auf $40^{\circ}$ ohne Alkoholzusatz niemals zu aktiven $R$. führte, während diese Temperatur bei gleichzeitiger Einwirkung von Alkohol die weitaus günstigsten Resultate lieferte.

Es scheint also zur Darstellung des H.-A. ein gewisses MittelmaB einer auf verschiedene Weise zu erzielenden chemischen Erschütterung des kolloidalen Ausgangsmateriales nötig zu sein. Ist diese Erschütterung zu gering, so kommt es gar nicht zur Bildung des aktiven Umwandlungsproduktes, ist sie zu stark, so gelangt man gleich zu den im Reagensglas indifferenten Derivaten, wie sie ja auch bei der spontanen Zersetzung eines aktiven $R$. sich bilden.

Was nun die Wirkung des zur Vakuumdarstellung verwendeten Alkohols anlangt, so wurden auch darüber mannigfache Parallelversuche angestellt, von denen hier nur mitgeteilt werden möge, daß bei der Erhitzung auf $80^{\circ}$ mit Alkohol wirksamere Rückstände in kürzerer Zeit erhalten wurden, als obne dieses Reagens, daB aber auch hier die Darstellung im Vakuum bei $40^{\circ}$ die besten Ergebnisse lieferte. Auch dieses Resultat scheint in dem oben angedeuteten Sinne zu sprechen, daB nämlich ein und dieselbe Schädlichkeit je nach der Dauer und Intensität ihrer Einwirkung die Entstehung des H.-A. begünstigt, aber auch zur Bildung der inaktiven Modifikation AnlaB geben kann.

Weiterhin wurde in der Hoffnung, die doch unbequeme Valsuum. destillation vermeiden zu bönnen, die Wirkung anderer Agentien versucht. In Anlehnung an die Versuche von Weichardt über sein Ermüdungstoxin, in denen es ihm gelungen war, aus an sich ungiftigen EiweiBkörpern durch Erschütterung des Moleküles anf chemischem und elektrolytischem Wege giftige Körper zu erhalten, wurde versucht, ob nicht auch hier durch die Einwirkung oxydierender oder reduzierender Mittel oder durch den elektrischen Strom das Agglutinationsphänomen in die Erscheinung trete. Die hierher gehörigen Versuche wurden ganz 
analog jenen Weichardts ausgeführt, je zweimal wiederholt, dann aber, da sie durchgehends negative Resultate lieferten, abgebrochen. E's gelang also auf den angegebenen Wegen bisher nicht, zu agglutinierenden Harnen zu gelangen.

Die mitgeteilten Versuche insgesamt scheinen in Zusammenhalt mit den unveröffentlichten Ergebnissen von H. Eppinger und Pregl dafür zu sprechen, daß für die große Verschiedenheit der Aktivität verschiedener unter scheinbar denselben Versuchsbedingungen gewonnener R. nicht so sehr essentielle Unterschiede in der Menge der kolloidalen Vorstufe, als vielmehr nicht vermeidbare Differenzen in der Darstellungsmethodik verantwortlich gemacht werden müssen. Es mag sein, daß schon geringe Unterschiede in dem Säuregrad und nicht zu umgehende Schwankungen in der Darstellungszeit die Ursache der in Rede stehenden Inkonstanz des agglutinierenden Titres abgeben.

Daneben spielt aber noch ein zweiter Faktor eine wichtige Rolle, auf den im IV. Abschnitte näher eingegangen werden soll: Die gleichzeitige Bildung einer die Reaktion hemmenden Modifikation.

Noch viel schwankender als die Ergebnisse der Vakuumdestillation waren jene der Einwirkung höherer Hitzegrade auf kleinere Harnmengen offenbar deshalb, weil hier die oben als maßgebend erkannten Einflüsse noch viel weniger exalit bewertet werden können. Ich bin deshalb in den letzten Monaten, wenn es darauf ankam, R. starker Wirkung zu erhalten, von der Darstellung bei $80^{\circ}$ ganz abgekommen. Es hat sich mir die folgende Darstellungsmethode als die brauchbarste erwiesen:

$500^{\mathrm{cm}}$ genuinen Morgenharnes werden zu gleichen Teilen mit absolutem Alkohol versetzt. Es kann nun von dem sich abseheidenden Niedersehlag abfiltriert werden oder aber die getrübte Flüssigkeit im Vakuum bei einer $40^{\circ}$ nicht übersteigenden Temperatur beiläufig bis zur Sirupdicke eingeengt werden. Der Rückstand wird in etwas Wasser aufgenommen, klarfiltriert, mit Kalilauge sofort und exakt neutralisiert, da sonst die freie Säure eine störende Hämolyse hervorrufen würde. Nun wird auf das Volumen von $1^{\mathrm{com}}$ der Lösung $=5^{\mathrm{cem}}$ des Ausgangsmateriales aufgefüllt. Der Gehalt an Harnsalzen stört die Beobachtung des Agglutinationsphänomens nicht, doch können diese durch eine 24- bis 48 stündige Dialyse so weit entfernt werden, daB sie praktisch nicht mehr in Betracht kommen. Der großen Hinfälligkeit der agglutinierenden Wirkung und der eintretenden Verdünnung wegen empfiehlt es sich, bei Anwendung der Dialyse von einer Lösung der Konzentration von 1:10 auszugehen, da durch eine neuerliche Einengung des Dialysates, die ja unter Alkoholzusatz erfolgen muß, die agglutinierenden Eigenschaften, wenn auch in den meisten Fällen nicht vollständig zerstört, so doch schwer geschädigt 
werden. Gewicht ist zu legen auf die sofortige Neutralisation der Lösungen, da sonst in kurzer Zeit ihre Wirksamkeit ganz außerordentlich abnimmt. Weil viele der so gewonnenen $R$. innerhalb der ersten 24 Stunden nach der Darstellung die im Abschnitt IV näher zu analysierende Hemmungszone oft so stark ausgebildet zeigen, daB ein H.-A. fast vollständig verdeckt sein kann, innerhalb dieser Zeit aber das sie bedingende Agglutinoid meistens spontan zugrunde geht, neue Mengen dieses aber bei der Zersetzung des Agglntinins nicht gebildet werden, also die Wirksamkeit eines R. erst nach dem angegebenen Zeitraum richtig erkannt zu werden vermag, so empfiehlt es sich, in allen Fällen, wo Titrebestimmungen vorgenommen werden, dies erst am 2. Tag und wiederholt zu tun. Erst dann bekommt man ein richtiges Bild von der Wirksamkeit eines Rückstandes. Als Beleg hierfür sei auf den in der ersten Mitteilung, Tabelle XII, zitierten Versuch neuerdings hingewiesen.

Was die Reindarstellung des agglutinierenden Harnkörpers anlangt, so wurde wiederholt versucht, ihn aus $R$. durch weitgehende Dialyse und nachheriges Aussalzen mit Ammonsulfat wenn auch nur annähernd rein zu gewinnen. Es zeigte sich in solchen Versuchen immer wieder, daß durch den chemischen Eingriff die Wirksamkeit eine derartig schwere Schädigung erfuhr, daB endlich unwirksame Lösungen erhalten wurden. Dabei konnte aber, wie aus dem nachfolgenden Versuche hervorgeht, wenigstens festgestellt werden, daB Ammonsulfat den Körper zu fällen vermag.

Versuch: Es wird ein vorher in seiner Wirksamkeit austitrierter $\mathbf{R}$. nach vorausgehender Dialyse bis zur Sättigung mit Ammonsulfat ausgesalzen. Der auf dem Filter gesammelte, abgeprelite und durch Dialyse von dem Fällungsmittel fast vollständig befreite Niederschlag besitzt ein deutliches Agglutinationsvermögen, das Filtrat vom Ammonsulfatniederschlag hingegen, welches auf dieselbe Weise behandelt worden war, nicht. Es ist eine sehr starke Abnahme des agglutinierenden Titres gegenüber demjenigen einer anderen R.-Probe zu bemerken, welche gleich lange dialysiert, hirgegen nicht mit Ammonsulfat behandelt worden war. Während für die aus dem Niederschlage gewonnene Flüssigkeit als kleinste agglutinierende Dosis $0.005^{\mathrm{ecm}}$ rechnerisch zu erwarten stand, zeigte sich nur mehr $0.5^{\mathrm{cm}}$ als wirksam.

Diese hohe Labilität ist es auch, die bis heute den Versuch einer chemischen Identifizierung mit dem von Abderhalden und Pregl beschriebenen EiweiBablömmling als aussichtslos erscheinen lieB. 
III.

Im Hinblick auf später zu erwähnende Ergebnisse war es von besonderem Interesse, über die hemmende Wirkung von EiweiB- bzw. Serumlösungen Näheres zu erfahren und zu prüfén, wie sich andere Körper hinsichtlich ibrer Schutzwirkung verhalten.

Was zunächst Eiweißlösungen anlangt, so konnte früher schon ganz allgemein nachgewiesen werden (vgl. erste Mitteilung, Tabelle XV bis XVII!), daß sie ein intensives Hemmungsvermögen gegen die agglutinierende Wirkung der $R$. besitzen und $d a B$ sie unter Bildung einer Trübung, später eines flockigen Niederschlages mit ihnen reagieren, während unwirksame oder durch Erhitzen inaktivierte R. kein Fällungsvermögen gegen EiweiBlösungen besitzen. Diese Beobachtungen decken sich ebensowohl mit den von Landsteiner und Jagič bei anorganischen Kolloiden und bei Abrin als mit den ron Michaelis und Steindorf bei Ricin und Abrin erhobenen Befunden. Es muB betont werden, daB durch diesen F'ällungsvorgang sowohl die agglutinierende Kraft der aktiven Rückstände als auch die Schutzwirkung der KiweiBlösungen erschöpft wird, daß also beide Körper aufeinander unter Bildung eines indifferenten Niederschlages reagieren.

Im Hinblick darauf, daB die aktiven $R$. aus einem kolloidalen Körper indifferenter Natur sich bilden, wurde in der ersten Vlitteilung, wenn auch schon damals manche Erfahrungen in anderem Sinne sprachen, die Möglichkeit hervorgehoben, es könnte vielleicht gerade dieser indifferente Körper sein, der im Harn zur Ausscheidung gelangt und daB durch Zerstörung einer thermolabileren, neutralisierenden Komponente infolge des Eingriffes das H.-A. frei wird.

Sollte diese Möglichkeit an Wahrscheinlichkeit gewinnen, so muBte vorausgesetzt werden, daß auch der hemmende Körper der Eiweißlösungen thermolabil sei, daB es also durch Erhitzen von EiweiBlösungen gelänge, ihre schützende Kraft aufzuheben.

In orientierenden Vorversuchen konnte nun festgestellt werden, dab die hemmende Kraft von Eiweißlösungen keinerlei Einbuße erfährt selbst durch stundenlanges Verweilen bei $56^{\circ}$, Erfahrungen, die schon gegen die oben ausgesprochene Vermutung herangezogen werden mubten. In unten näher zu besprechenden Versuchen zeigte es sich aber auch weiterhin, daß selbst ein Kochen der Eiweißlösungen bei essigsaurer Reaktion ihre Schutzwirkung vollständig nicht zu zerstören vermochte.

Es interessierte daher, festzustellen, ob es denn die Eiweißkörper soleher Lösungen sind, an denen die Schutzwirkung haftet, oder ob hier die Lipoide des Serums in Betracht kamen. Darüber belehrte der folgende Versuch. 
Versuch: Bei $56^{\circ}$ inaktiviertes Rinderserum wird in dem Verhältnis von 1:5 mit Wasser verdünnt (Kolonne A der Tabelle). Dieses Ausgangsmaterial wird mit Essigsäure schwach angesäuert und auf dem Wasserbade so lange erhitzt, bis eine vollständige Fällung der unter diesen Versuchsbedingungen fällbaren Eiweißkörper eingetreten war. Der Niederschlag wird durch Filtration von der Flüssigkeit (Kolonne $\mathbf{B}$ der Tabelle) getrennt und diese zur Entfernung der Lipoide dreimal mit Äther bei schwach essigsaurer Reaktion ausgeschüttelt. Die ausgeschüttelte Flüssigkeit wird bis zur Verjagung des Äthers auf dem Wasserbade erhitzt (Kolonne C der Tabelle), der ätherische Auszug bei gelinder Wärme abgedunstet und der spärliche Rückstand in Kochsalzlösung in einem dem Ausgangsmateriale entsprechenden Mengenverhältnisse aufgenommen (Kolonne D der Tabelle). Nach Neutralisation der freien Säure in den einzelnen Proben werden diese an einem aktiven Rückstand auf ihre Schutzwirkung geprüft. Das Ergebnis dieses Versuches ist aus Tabelle IV zu entnehmen.

Tabelle IV.

\begin{tabular}{|c|c|c|c|c|c|c|c|c|c|}
\hline & \multicolumn{2}{|c|}{ A } & \multicolumn{2}{|c|}{ B } & \multicolumn{2}{|c|}{ C } & \multicolumn{2}{|c|}{ D } & \multirow[t]{2}{*}{$\mathrm{NaCl}$-Lösung } \\
\hline & 0.05 & 0.025 & 0.05 & 0.025 & 0.05 & 0.025 & 0.05 & 0.025 & \\
\hline 0.5 & d. & sd. & fm. & $\mathrm{m}$. & fm. & m. & m. & $\mathrm{m}$. & m. \\
\hline $0 \cdot 25$ & - & d. & sd. & $\mathrm{fm}$. & sd. & $\mathbf{m}$. & m. & m. & $\mathrm{m}$. \\
\hline 0.13 & - & - & d. & sd. & sd. & sd. & $\mathrm{m}$. & m. & m. \\
\hline 0.06 & - & - & - & - & - & Sp. & $\mathrm{m}$. & m. & m. \\
\hline 0.03 & - & - & - & - & - & - & $\mathbf{m}$ & m. & m. \\
\hline 0.015 & - & - & - & - & - & - & m. & $\mathrm{m}$. & $\mathrm{m}$. \\
\hline 0.007 & 一 & - & - & - & - & - & sd. & sd. & sd. \\
\hline 0.009 & - & - & - & - & - & - & Sp. & Sp. & Sp. \\
\hline 0.002 & - & - & - & - & - & - & - & - & - \\
\hline$\emptyset$ & - & - & - & - & - & - & - & - & - \\
\hline
\end{tabular}

Es zeigte sich also, daB die Entfernung der durch Essigsäure in der Hitze bei essigsaurer Reaktion fällbaren Eiweißkörper die schützende Kraft zwar etwas vermindert hatte, daß diese aber keineswegs vollständig verloren gegangen war. Ebenso erlitt durch die Ausschüttelung mit Äther die hemmende Kraft keine essentielle Einbuße, wie auch die in Wasser emulgierten Lipoide eine nennenswerte Schntzwirkung nicht zu entfalten vermochten. In zahlreichen parallelen Versuchen, die mit verschiedenen Serumarten und Lösungen von Hühnereiklar ausgeführt wurden, zeigte es sich gan\% allgemein, daß zwar meistens mit der Ausfällung der Eiweißkörper eine nachweisbare Abnahme aber kein vollständiger Verlust der Schutzwirkung eingetreten war, daß aber niemals durch die Ätheraussehüttelung der enteiweiBten Lösung eine Veränderung der Hemmungsintensität beobachtet werden konnte. Dagegen trat bei einer in derselben Weise behandelten Albuminlösung (aus Albumin Grübler gewonnen), die gleichfalls eine intensive Schutzwirkung äuBerte, nach der Fällung auf dem Wasserbade 
bei essigsauerer Reaktion ein vollständiger Verlust der Hemmung auf. Es scheint im Hinblick auf diese Ergebnisse nicht uninteressant, hervorzuheben, daB auch Peptonlösungen, die aus Pepton Witte gewonnen worden waren, gleichfalls unter Trübung mit den aktiven R. reagierten und eine intensive Schutzwirkung ausübten. Eine Lösung von Agar-Agar hingegen zeigte dieses $\mathrm{Phänomen}$ nicht.

Gegenüber diesen Befunden, gegenüber den Erfahrungen von Kyes und Sachs bei den Schlangengiften und jenen von Landsteiner und Jagič bei ihren anorganischen Kolloiden wurde geprüft, welchen Einfluß denn Leeithin und Cholestearin auf den in Rede stehenden Agglutinationsvorgang auszuüben vermögen. In zahlreichen Versuchen konnte in ganz gleicher Weise gefunden werden, daB beide Körper, in besonders hohem Maße aber das Lecithin, eine Schutzwirkung für die Erythrozyten besitzen.

Tabelle V.

\begin{tabular}{|c|c|c|c|c|c|c|c|}
\hline $\begin{array}{c}\text { Lecithin } \\
\text { in Milligrm.: }\end{array}$ & 0 & 0.13 & 0.065 & 0.037 & 0.0185 & 0.0097 & 0.0048 \\
\hline Rückstand: & & & & & & & \\
\hline $0 \cdot 5$ & m. & - & d. & $\mathrm{fm}$. & m. & m. & $\mathrm{m}$. \\
\hline 0.25 & $\mathrm{~m}$. & - & Sp. & d. & fm. & fm. & m. \\
\hline 0.13 & $\mathrm{~m}$. & - & - & - & sà. & fim. & $\mathrm{m}$ \\
\hline 0.06 & m. & - & - & - & Sp. & sd. & fm. \\
\hline $0 \cdot 03$ & fm. & - & - & - & - & Sp. & d. \\
\hline 0.015 & d. & - & - & - & - & - & - \\
\hline 0.007 & Sp. & - & - & - & - & - & - \\
\hline 0.004 & - & - & - & - & - & - & - \\
\hline$\emptyset$ & - & - & - & - & - & - & - \\
\hline
\end{tabular}

$\mathrm{Zu}$ dem in Kolonne I austitrierten R. werden die in den übrigen Kolonnen angegebenen Lecithinmengen in $0.5 \mathrm{~cm}$ Kochsalzlösung suspendiert hinzugegeben. Nach energischem Umschütteln werden die Erythrozyten zugefügt. Als Stammlösung diente eine 1 prozentige Lösung von Lecithin ab ovo Merck in reinstem Methylalkohol. Wurde die Versuchsanordnung in der Weise verändert, daß zuerst Erythrozyten und Lecithinemulsion gemischt dureh 1 Stunde bei $37^{\circ}$ gehalten und dann erst die aktiven $R$. hinzugefügt wurden, so konnte auch hier nur eine hemmende Wirkung des Lecithins beobachtet werden.

Es ergibt sich aus diesen Versuchen, daB im Gegensatz zu der komplettierenden Wirkung dieses Lipoides gegenüber der kolloidalen Kieselsäure und dem Cobragifte hier nur von einer die Reaktion hemmenden Eigenschaft die Rede sein kann. Endlich verdient hervorgehoben zu werden, daß in sehr stark wirksamen und konzentrierten R., wie dies Landsteiner und Jagič auch für die anorganischen Kolloide beschrieben haben, eine Ausflockung der Emulsion in die Erscheinung trat. 
IV.

Es wurde in der ersten Mitteilung des eigentümlichen Umstandes Erwähnung getan, daß manche $R$, gleich nach ihrer Darstellung geprüft, in konzentrierten Lösungen keine Agglutination hervorriefen, während in verdünnteren die Reaktion ungehindert ablief. Untersucht man solche R. nach einiger Zeit wieder, so tritt nun dieses Phänomen nicht mehr in die Erscheinung, gleichzeitig ist aber auch der Agglutinationstitre, also die oberste noch wirksame Verdünnungsgrenze nicht unbeträchtlich hinausgerückt. Der Wichtigkeit dieser Beobachtung für die Deutung der in Rede stehenden Erfahrung wegen sei hier der in der ersten Mitteilung in Tabelle VII zitierte Versuch nochmals wiederholt.

Tabelle VI.

\begin{tabular}{|c|c|c|c|c|}
\hline \multirow{2}{*}{$\begin{array}{l}\text { M e n g e } \\
\text { in } 1 \mathrm{cem}\end{array}$} & \multicolumn{4}{|c|}{ Rüekstand geprüft nach der Darstellung: } \\
\hline & sofort & $\begin{array}{l}6 \text { Standen } \\
\text { später }\end{array}$ & $\begin{array}{l}28 \text { Stunden } \\
\text { später }\end{array}$ & $\begin{array}{l}48 \text { Stunden } \\
\text { später }\end{array}$ \\
\hline $1 \cdot 0$ & - & $\mathrm{m}$. & m. & $\mathrm{m}$. \\
\hline $0 \cdot 5$ & - & m. & ni. & $\mathbf{n}$. \\
\hline 0.25 & - & $\mathrm{n}$. & m. & m. \\
\hline $0 \cdot 13$ & Sp. & m. & $\mathrm{m}$. & in. \\
\hline 0.06 & fm. & m. & m. & $\mathrm{m}$. \\
\hline $0 \cdot 03$ & m. & w. & $\mathrm{m}$. & 1rı. \\
\hline 0.015 & m. & fm. & $\mathrm{m}$. & m. \\
\hline 0.007 & d. & d. & $\mathrm{m}$. & in. \\
\hline 0.004 & - & Sp. & in. & m. \\
\hline 0.002 & - & - & fin. & fm. \\
\hline 0.001 & - & - & - & d. \\
\hline 0.0005 & - & - & - & $\ldots$ \\
\hline 0.00025 & - & - & - & - \\
\hline$\emptyset$ & - & - & - & - \\
\hline
\end{tabular}

In der überwiegenden Zahl der Fälle wurde jedoch damals dieses Phănomen nicht beobachtet. Seither konnte aber, vielleicht infolge erhöhter Übung in der Darstellung, vielleicht infolge gesteigerter Aufmerksamkeit an ganz frischen $R$. regelmäBig diese Beobachtung gemaeht werden.

Es wurde damals bei Annahme einer haptophoren und einer labileren agglutinophoren Gruppe unter den verschiedenen Erklärungsmöglichkeiten besonders darauf hingewiesen, daB sich vielleicht die folgende Deutung als die wahrscheinlichste werde vertreten lassen: Besetzung der vorhandenen Erythrozyten durch ein Agglutinoid, welches mit dem Verluste seiner agglutinophoren Gruppe eine Steigerung seiner Avidität erfahren hat und 
nun die Besetzung dẹ Blutkörperchen durch das weniger avide Agglutinin hindert.

Es wurde ferner der Umstand hervorgehoben, daB bei späteren Untersuchungen auch in den konzentrierten Lösungen Agglutination ungehindert eintrat und dabei eine erhebliche Steigerung des Agglutinationstitres sich bemerkbar machte.

Um nun das Phänomen der Hemmung mit dieser eben erwähnten sekundären Zunahme in Übereinstimmung zu bringen, konnte nur angenommen werden, daB sich die hemmende Modifikation ausschlieBlich bei der Darstellung und gleichzeitig mit dem Agglutinin bildet, später aber, da die Bedingungen für ihre Entstehung nicht mehr gegeben sind, auch nicht mehr gebildet wird, vielmehr spontan zugrunde geht, während nun das gesamte, ursprünglich in der Lösung enthaltene Agglutinin zur Wirksamkeit gelangt.

Es haben nun Landsteiner und Jagič auch für ihre anorganischen Kolloide eine Hemmung oder Verzögerung der Reaktion dureh Überschuß wirksamer Substanz beschrieben, erwähnen aber leider nichts darūber, ob in älteren Lösungen die Erseheinung versehwindet, namentlich aber, ob dann ein Hinausrücken des agglutinierenden Titres zu beobachten ist. Besonders dieser letztgenannte Umstand scheint mir zur richtigen Deutung der in Rede stehenden Tatsachen von Wichtigkeit zu sein. Man könnte bei der Labilität des agglutinierenden Kïrpers den alleinigen Wegfall der Hemmungszone in älteren Lösungen ohne Erhöhung des agglutinierenden Titres' sehr wohl erklären durch das Vorhandensein und ein darauf folgendes spontanes Zugrundegehen eines die Reaktion hemmenden Überschusses agglutinierender Substanz. Die Erscheinung der Zerstörung der hemmenden Zone bei gleichzeitiger Erhöhung des Titres ist aber schlechterdings nicht anders als durch das Verschwinden eines lediglich hemmenden und durch das Erhaltenbleiben eines agglutinierenden Körpers denkbar. Wollte man auch hier ausschlieBlich einen agglutinierenden Körper supponieren, dessen Überschuß die Hemmung bewirke, so wäre nicht einzusehen, warum in diesem Fall mit dem Verluste der hemmenden Zone eine Titreerhöhung eintritt, da ja doch einfach ein in konzentrierten Lösungen hemmender Übersehuß nun beseitigt ist, die obere Grenze der Wirksamkeit aber auch anfangs schon erreicht sein mußte.

Es scheint mir also das Hinausrücken des Titres bei gleichzeitigem Verluste der Hemmungszone schon der Beweis für eine die Reaktion hemmende Modifikation des aktiven H.-A. zu sein. Immerhin war es aber wertroll, dafür und namentlich für die höhere Avidität des Agglutinoides weitere Beweise zu erbringen. Diese lieferte ein genaueres Studium der Wirkung hemmender Körper gegen derartige $R$. 
Prüft man den Einfluß einer Lecithinemulsion auf einen, die Hemmungszone zeigenden $R$., wie aus Tabelle VII herrorgeht, so zeigt es sich, daß es neben der im dritten Abschnitte beschriebenen hemmenden Wirking, die sich in einer Verkürzung der Reaktionsbreite zu erkennen gibt, auch zu einer Aufhebung der Hemmungszone in den konzentrierteren Lösungen koinmt. Diese Erscheinung wird besonders deutlich, wenn man den zeitlichen Ablauf der Reaktion berücksichtigt. Es kommt schon nach kurzer Zeit in solchen Röhrchen zu stürmischer Agglutination, welche ohne Lecithinzusatz überhaupt noch keine merkbare Veränderung der Erythrozyten erkennen lassen. Mit fallenden Lecithinmengen aber vermindert sich die Hemmung der oberen noch wirksamen Verdünnungsgrenze in demselben $\mathrm{MaBe}$, wie die Hemmungszone dann deutlich wieder zum Vorschein kommt.

Tabelle VII.

\begin{tabular}{|c|c|c|c|c|c|c|c|c|c|}
\hline \multirow{2}{*}{$\begin{array}{l}\text { Rückstand } \\
\text { in } 1^{\mathrm{ccm}}\end{array}$} & \multicolumn{8}{|c|}{ Mengen des zugesetzten Lecithins in Milligrammen } & \multirow{2}{*}{ NaCI-Lösung } \\
\hline & 0.5 & 0.25 & 0.13 & $0 \cdot 06$ & 0.03 & 0.015 & 0.008 & 0.009 & \\
\hline $1 \cdot 0$ & m. & $\mathbf{m}$. & m. & fm. & sd. & d. & d. & d. & d. \\
\hline 0.5 & d. & m. & m. & m. & fm. & fm. & fm. & fm. & sd. \\
\hline $0 \cdot 25$ & Sp. & d. & fm. & m. & m. & m. & m. & $\mathrm{m}$. & $\mathrm{m}$ \\
\hline 0.13 & - & Sp. & sd. & m. & $\mathrm{m}$. & $\mathrm{m}$. & $\mathbf{m}$ & $\mathrm{m}$. & $\mathrm{m}$ \\
\hline $0 \cdot 06$ & - & - & - & sd. & fm. & fm. & fm. & $\mathbf{m}$. & m. \\
\hline 0.03 & - & - & - & Sp. & d. & sd. & d. & fm. & sd. \\
\hline 0.015 & - & - & - & - & - & d. & Sp. & sd. & d. \\
\hline 0.007 & - & - & - & - & - & - & - & d. & Sp. \\
\hline 6 & - & - & - & - & - & - & - & - & - \\
\hline
\end{tabular}

Wenn man die früher angestellten Überlegungen berücksichtigt, so wäre eine doppelte Deutung dieser Resultate möglich: 1. Bei der Annahme, die Hemmungszone werde lediglich hervorgerufen durch einen ÜberschuB des H.-A.: Bindung des Überschusses in den konzentrierten Lösungen und dadurch Beschleunigung und Verstärkung der Reaktion im Bereiche der Hemmungszone bei gleichzeitiger Verschmälerung der Reaktionsbreite. 2. Bei der Annahme, die Hemmungszone werde durch eine die Erythrozyten zwar besetzende, sie aber nicht mehr agglutinierende Modifikation des H.-A. erzeugt: Das Agglutinoid besitzt ebenso wie gegen die Blutkörperchen, so auch gegen das Lecithin eine höhere Avidität als das Agglutinin. Dadureh Wegfall der hemmenden Zone in konzentrierteren Lösungen und bei entsprechend abgestuftem Lecithinzusatz Beschleunigung der Reaktion deshalb, weil nun dort das verfügbare Agglutinin zur Wirkung kommen kann. Denn das avidere Agglutinoid hat sich mit dem hemmenden Lecithin verbunden, beide Körper haben sich gegenseitig in 
ihrer Wirkung paralysiert. Bei fallenden Mengen der R. und bei konstantem Lecithinzusatz kommt es zu einer Hemmung in der obersten noch wirksamen Verdünnungsgrenze, da in dieser Lecithin im ÜberschuB vorhanden ist und hier nicht nur das Agglutinoid, sondern auch das Agglutinin zu hemmen vermag.

Der angeführte Versuch gestattet es also nicht, zwischen beiden Möglichkeiten exakt zu entscheiden, wohl aber ein nunmehr anzuführendes Ergebnis:

Dieser Versuch betrifft, wie aus Tabelle VIII hervorgeht, einen R., der kurz naeh der Darstellung fast unwirksam sich zeigte. In der Menge von $0.015^{\mathrm{cem}}$ war eine spurenweise Agglutination zu bemerken, konzentriertere oder auch verdünntere Lösungen erwiesen sich als völlig unwirksam. Wurden nun, wie aus der Tabelle ersichtlich ist, zu konstanten Versuchsreihen fallende Lecithinmengen hinzugefügt, so konnte das Auftreten stürmischer Agglutination und ein gleichzeitiges Hinausrücken der wirksamen Versuchsmengen über die obenangeführte Grenze der spurenweisen Wirksamkeit verzeichnet werden.

Tabelle VIII.

\begin{tabular}{|c|c|c|c|c|c|}
\hline \multirow{2}{*}{$\begin{array}{l}\text { Rückstand } \\
\text { in } 1 \text { @em }\end{array}$} & \multicolumn{5}{|c|}{ Mengen des zugesetzten Lecithins in Milligrammen } \\
\hline & 0.25 & 0.13 & 0.06 & 0.03 & 0 \\
\hline $1 \cdot 0$ & - & - & - & $\cdots$ & - \\
\hline 0.5 & $\mathrm{~m}$. & Sp. & - & - & - \\
\hline 0.25 & m. & $\mathrm{m}$. & sd. & - & - \\
\hline 0.13 & $\mathrm{fm}$. & $\mathrm{m}$. & m. & d. & - \\
\hline 0.06 & - & d. & m. & $\mathrm{m}$. & - \\
\hline 0.03 & - & - & $\mathrm{fm}$. & $\mathrm{m}$. & - \\
\hline 0.015 & - & - & d. & m. & Sp. \\
\hline 0.007 & - & - & - & sd. & - \\
\hline$\emptyset$ & - & - & - & - & - \\
\hline
\end{tabular}

Dieses Ergebnis ist, wie ich glaube, nicht anders als in dem Sinne $z u$ deuten, es werde die hemmende Zone frischer Rückstände durch das Nebeneinander und durch die Konkurrenz eines gegen die Erythrozyten und gegen das Leeithin avideren Agglutinoides mit einem weniger aviden Agglutinin verursacht. Der in Tabelle VIII beobachtete $R$. zeigte übrigens, einige Zeit später untersucht, einen vollständigen Verlust der Hemmungszone und eine kräftige Agglutination innerhalb von Verdünnungsgrenzen, die weit über die in Kolonne I angegebenen hinausgingen.

Wenn man diese Ergebnisse mit jenen in der ersten Mitteilung angeführten vergleicht, welche beweisen, daB es gelingt nach Vorbehandlung eines aktiven $R$. mit Blutkörperchen das H.-A. elektiv zu binden, so wird man zu der Auffassung gedrängt, der hier in Rede stehende Körper besitze zwei voneinander differente Gruppen, eine stabilere, in 
gewisser Hinsicht haptophore und eine labilere agglutinophore Gruppe, er verhalte sich also ähnlich wie ein Rezeptor zweiter Ordnung im Sinne Ehrlichs. Wie aber diesen recht eindeutigen Resultaten gegenüber die Beobachtung erklärt werden müsse, $d a b$ es bis jetzt weder durch intraperitoneale noch durch subkutane Vorbehandlung von Tieren (Kaninchen) gelungen ist, Antikörper zu erzeugen, darūber können nur weitere eingehende Versuche Aufschlub geben. Meiner Meinung nach müBten sich solche Arbeiten vor allem mit ausgedehnten Immunisierungsversuchen auf jedem der heute gebräuchlichen Wege beschäftigen und dann zur Beantwortung der Frage übergehen, an welchem Teile der Erythrozyten die aktiven R. angreifen. Es könnte vielleicht die Entscheidung, ob das Lecithin der Stromata die agglutinierende Wirkung vermittele, hier eine Erklärung für die erwähnten Widersprüche bringen.

Die „amphotere“ Wirkung des Lecithins gegen R. mit Hemmungszone teilen alle die Reaktion hemmenden Körper, also Eiweißlösungen, Pepton ${ }^{1}$ und Cholestearin.

Diese Eigenschaft gestattet es außerdem über die im zweiten $A b-$ schnitte besprochene Inkoustanz des Agglutinationstitres verschiedener $R$. teilweise Aufklärung zu erhalten. Während früher kurz nach der Darstellung untersuchte und als unwirksam gefundene $R$. im allgemeinen als solche registriert und nicht weiter beachtet wurden, gestattete eine Benützung der eben erwähnten Beobachtungen, zu prüfen, ob denn nicht das Fehlen der agglutinierenden Wirkung auf eine starke Agglutinoidbildung neben einer in untergeordnetem Maße nehenhergehenden Entstehung der altiven Modifikation zurückgeführt werden müsse. Für eime Reihe solcher, namentlich durch Erhitzen anf $80^{\circ}$ hergestellter $\mathrm{R}$. gelang es nun tatsächlich, durch einen vorsichtig abgestuften Zusatz von Lecithin daron sich zu überzeugen, daß in ihnen das wirksame H.-A. nicht etwa gar nicht gebildet, sondern einfach durch das Agglutinoid überdeclit war. Eine detaillierte Angabe dieser Versuche scheint überflüssig, da Tabelle VIII ein derartiges Paradigma enthält. Es muß aber darauf hingewiesen werden, daB eine Schwankung des Titres nur zum Teil auf diese Ursachen zurückgeführt werden darf und $\mathrm{da} B$ wiederholt auch $R$. erhalten wurden, welche wirklich unwirksam waren.

Es drängte sich weiterhin die Frage auf, ob nicht etwa das weder hemmende noch agglutinierende Ausgangsmaterial des genuinen Harnes seine Eigenschaft einem äquilibrierten Nebeneinander von Agglutinoid

1 Einer privaten Mitteilung K. Iandsteiners entnehme ich übrigens, dab dieser Autor kürzlich eine hemmende Wirkung von Pepton auf die Abrinagglutination beobachtet hat. 
und Agglutinin verdanke, was ja durch die oben besprochene Versuchsanordnung leicht $\mathrm{zu}$ entscheiden war. Derartige Versuche, genuinen Harn durch vorsichtig abgestuften Lecithinzusatz zu aktivieren, fielen aber durchaus negativ aus.

Im Hinblick auf frühere Erklärungsversuche über den späteren Wegfall der Hemmungszone bei längerem Aufbewahren der R. mußte geprüft werden, ob es bei der Zersetzung des Agglutinins unter verschiedenen Versuchsbedingungen nicht zur Bildung der hemmenden Modifikation komme. Die Technik dieser Versuchsreihen gestaltete sich sehr einfach. Es wurden teils spontan unwirksam gewordene, teils durch verschiedene thermische und chemische Einflüsse inaktivierte R. auf ihre hemmende Wirkung an aktiven $R$. in versehiedenen Versuchsmengen geprüft. Dabei konnte die früher gemachte Erfahrung vollauf bestätigt werden, daß weder bei der spontanen Zersetzung, noch bei der Inaktiviernng bei 60,80 und $100^{\circ} \mathrm{C}$ jemals die hemmende Modifikation wahrgenommen wurde. Es scheint sich diese nur bei der Entstehung des Agglutinins und zugleich mit diesem aus der kolloidalen und indifferenten Vorstufe, nicht aber aus der aktiven Modifikation zu bilden.

Zusammenfassend möchte ich endlich hervorheben, daß es durch verschiedene thermische und chemische Eingriffe gelingt, aus einer möglicherweise mit dem von Schattenfroh dureh serologische, von Abderhalden und Pregl auf ehemischem Wege nachgewiesenen und als EiweiBabkömmling definierten, im Reagenzglasversuche indifferenten Körper kolloidaler Natur gelingt, eine stark agglutinierende Modifikation zu erhalten. Häufig bildet sich aber daneben gleichzeitig eine zweite, nicht nur unwirksame, sondern den Ablauf der Reaktion hemmende Zustandsform, die, labilerer Natur, rascher zugrunde geht als der aktive Körper. Dieser vermag jedoch, soweit heute die Erfahrungen reichen, bei seiner Zersetzung in diese hemmende Modifikation nicht überzugehen, sondern lediglich einen indifferenten Körper zu bilden. Trotz weitgehender Analogien mit gewissen Antigenen konnten bis heute durch Vorbehandlung von Kaninchen mit aktiven $R$. Immunisierungsprodukte nicht erhalten werden.

Diesen Tatsachen gegenüber muB besonders auf die Befunde Weichardts über die Bildung giftiger, mit seinem Ermüdungstoxin identifizierter Antigene hingewiesen werden. Es mag sein, daB es sich dort und hier um prinzipiell denselben Vorgang handelt. 
508 Hermann Pfeiffer: Agglutinierende Wirkung usw.

\section{Literatur-Verzeichnis.}

H. Pfeiffer, Diese Zeitschrift. 1906. Bd. LIV.

Landsteiner und Jagie, Münchener med. Wochenschrift. 1903. Nr. 18.

Dieselben, Ebenda. 1904. Nr. 27.

Dieselben, Wiener klin. Wochenschrift. 1904. Nr. 3.

Landsteiner, Zeitschrift für physikal. Chemie. 1905. Bd. LI. S. 741.

Landsteiner mit Uhlirz, Reich, v. Eisler und Stankovič in verschiedenen im Centralblattf. Bakteriologie, Bd. XXXIX-XLII erschienenen Arbeiten.

A. Siegfried, Archives internation. de Pharmakodynamie etc. 1901. T. IX.

Michaelis und Steindorf, Biochemische Zeitschrift. Bd. II. 43.

Preston Kyes, Berliner klin. Wochenschrift. 1902. Nr. 38-39.

Preston Kyes und Sachs, Ebenda. 1902. Nr.2-4.

Preston Kyes, Ebenda. 1903. Nr. 42-43.

Sehattenfroh, Archiv fior Bygiene. 1902. Bd. XLIV. S. 339.

Abderhalden und Pregl, Hoppe-Seylers Zeitschrift für physiol. Chemie. Bd. XLVI.

Weichardt, Serologische Studien usw. Monographie. Stuttgart 1906. 